\title{
Airway neurofibroma
}

\author{
Kamath Sriganesh, DM • Dhritiman Chakrabarti, MD - Tanmay Jadhav, MD • \\ Sudhir Venkataramaiah, MD
}

Received: 27 May 2015/Accepted: 3 June 2015/Published online: 11 June 2015

(C) Canadian Anesthesiologists' Society 2015

A 49-yr-old male with a known diagnosis of neurofibromatosis type 1 (NF-1) was scheduled for excision of a recurrent cerebellopontine angle neurofibroma. Previous anesthetics were uneventful, including airway assessment and management nine years earlier for removal of the original cerebellopontine angle neurofibroma and a cervical intradural extramedullary lesion. Magnetic resonance imaging in the sagittal and coronal planes showed a neurofibroma in the left cerebellopontine angle (dotted black arrow) (panel $A$ ) and on the right and left side (black arrows) in the patient's neck (panel B). Despite the anticipated difficult airway, the patient would not consent to awake intubation so with a difficult airway cart immediately available, succinylcholine was used to facilitate intubation after anesthesia induction with propofol. Direct laryngoscopy revealed a Cormack-Lehane grade 2 view, and there were bilateral posterior bulges of tissue into the supraglottic region extending into the midline (black arrows) (panel $C$ ). Both the tracheal intubation - performed with an 8.0-mm internal diameter endotracheal tube (ETT) - and the surgery were subsequently uneventful.

Neurofibromas are nerve sheath tumours and the most frequent tumours of neural origin. Their presence is essential for the diagnosis of NF-1, which is a hereditary disease of autosomal dominant inheritance occurring in one in every 3,000 births. ${ }^{1}$ There are two types of neurofibromas, dermatomal and plexiform. Plexiform neurofibromas are mostly congenital, with $50 \%$ occurring in the regions of the head, neck, face, and larynx with high rates of recurrence. ${ }^{2}$ Their presence in the neck region can pose a risk to the airway and make intubation difficult. Though awake intubation is recommended in these situations, ${ }^{3}$ our patient would not consent to it; consequently, we administered succinylcholine after explaining the procedure and confirming mask ventilation after induction of anesthesia. Surprisingly, intubation was not as difficult as anticipated and the passage of the ETT displaced the neurofibromas laterally. 

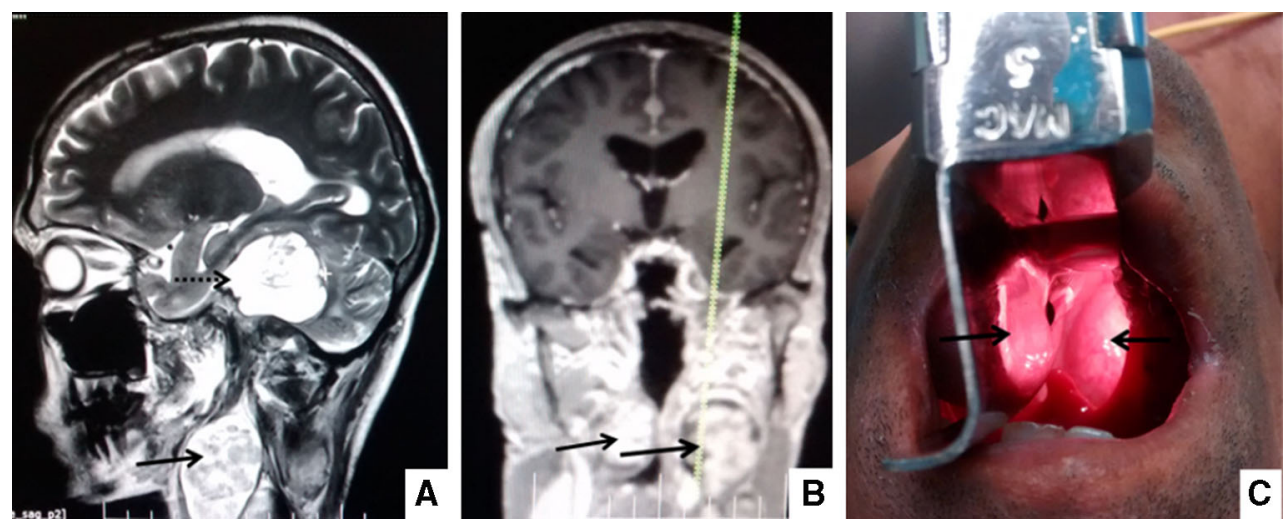

Figure Neurofibromas of the upper airway (A) Magnetic resonance imaging (MRI) (sagittal plane) showing neurofibromas in the left cerebellopontine angle and upper part of the left neck; (B) MRI

\section{Conflict of interest None declared.}

Funding None.

\section{References}

1. Shekar V, Rangdhol V, Baliah WJ, Thirunavukarasu S. An unusual oral manifestation of type 1 neurofibromatosis: a case report and review of literature. J Nat Sci Biol Med 2015; 6: 261-3. (coronal plane) showing bilateral neurofibroma in the upper neck obstructing the airway; (C) Direct laryngoscopic view during intubation showing two bulges on either side of the upper airway

2. Wise JB, Cryer JE, Belasco JB, Jacobs I, Elden L. Management of head and neck plexiform neurofibromas in pediatric patients with neurofibromatosis type 1. Arch Otolaryngol Head Neck Surg 2005; 131: 712-8.

3. Law JA, Broemling N, Cooper RM, et al. The difficult airway with recommendations for management - part 2 - the anticipated difficult airway. Can J Anesth 2013; 60: 1119-38. 